\title{
80 MUCOSAL HEALING IN PATIENTS TREATED WITH INFLIXIMAB IN CROHN'S DISEASE AND ULCERATIVE COLITIS
}

J Boyd, L Lee, S Lanzon-Miller Department of Gastroenterology, Milton Keynes General Hospital, UK

\subsection{6/gutjnl-2013-305143.80}

Aims/Background Infliximab (IFX) is a chimeric monoclonal antibody effective for inducing and maintaining remission in Crohn's disease (CD) and ulcerative colitis (UC). The efficacy of IFX in achieving clinical remission has been well demonstrated. However, data regarding mucosal healing based on duration of therapy is limited.

Method A retrospective cohort study of patients with CD and UC who had been treated with IFX at Milton Keynes General hospital. Patients were identified from the Planned Care Unit "infliximab diary" and their records were reviewed. Patient details, duration of therapy and results of endoscopic investigations were extracted into a standardized form. Mucosal healing was defined as no active inflammation on endoscopy. Patients who underwent a colonoscopy or sigmoidoscopy within one year of their last IFX dose were included in the study.

Results 21 patients (10 male) were studied with a median age of 37 years (range: $20-68$ years).

CD $(17 / 21)$

$\%$ ileal disease $12 \%(2 / 17)$

$\%$ ileocolonic $76 \%(13 / 17)$

$\%$ colonic disease $12 \%(2 / 17)$

UC (4/21)

$\%$ colonic disease $100 \%(4 / 4)$

4 patients had induction therapy at 0,2 and 6 weeks only. In this cohort mucosal healing was achieved in 50\% [(2/4) both CD].

17 patients had induction therapy followed by 8 -weekly maintenance infusions. The median duration of therapy was 24 months (range: 5-62 months). In this cohort mucosal healing was achieved in 29\% (5/17).

Conclusion IFX was successful in achieving mucosal healing for a high proportion of patients. The proportion of patients who had mucosal healing immediately after induction therapy of IFX was not statistically significant from the proportion of patients who had mucosal healing with maintenance therapy. This may be representative of the different disease characteristics of the two groups and we aim to investigate this further with a larger sample. 